\title{
Novel Copper Fluoride Analogs of Cuprates
}

\author{
Nikita Rybin, ${ }^{1}$ Dmitry Y. Novoselov, ${ }^{1,2,3}$ Dmitry M. Korotin, ${ }^{1,2}$ Vladimir I. Anisimov, ${ }^{1,2,3}$ and Artem R. Oganov ${ }^{1}$ \\ ${ }^{1}$ Skolkovo Institute of Science and Technology, 30 Bolshoy Boulevard, bld. 1, Moscow 121205, Russia \\ ${ }^{2}$ M. N. Mikheev Institute of Metal Physics of Ural Branch of Russian Academy of Sciences, \\ 18 S. Kovalevskaya St., Yekaterinburg 620137, Russia \\ ${ }^{3}$ Department of Theoretical Physics and Applied Mathematics, \\ Ural Federal University, 19 Mira St., Yekaterinburg 620002, Russia
}

(Dated: August 31, 2020)

\begin{abstract}
On the basis of the first-principles evolutionary crystal structure prediction of stable compounds in the $\mathrm{CuF}$ system, we predict two experimentally unknown stable phases $-\mathrm{Cu}_{2} \mathrm{~F}_{5}$ and $\mathrm{CuF}_{3} . \mathrm{Cu}_{2} \mathrm{~F}_{5}$ comprises two interacting magnetic subsystems with the $\mathrm{Cu}$ atoms in the oxidation states +2 and +3 . $\mathrm{CuF}_{3}$ contains magnetic $\mathrm{Cu}^{3+}$ ions forming a lattice with the antiferromagnetic coupling. We showed that some or all of $\mathrm{Cu}^{3+}$ ions can be reduced to $\mathrm{Cu}^{2+}$ by electron doping, as in the well known $\mathrm{KCuF}_{3}$. Significant similarities between the electronic structures calculated in the framework of $\mathrm{DFT}+\mathrm{U}$ suggest that doped $\mathrm{CuF}_{3}$ and $\mathrm{Cu}_{2} \mathrm{~F}_{5}$ may exhibit high- $T_{\mathrm{c}}$ superconductivity with the same mechanism as in cuprates.
\end{abstract}

Transition metal fluorides have been thoroughly studied during the last century [1, 2]. Among them coinage metal fluorides recently attracted considerable attention: $\mathrm{CuF}$ system in the electrochemistry field [3-6], $\mathrm{Ag}-\mathrm{F}$ as a potentially new route to superconductivity [7, 8], and Au$\mathrm{F}$ due to the unusual oxidation state of gold [9]. Actually, $\mathrm{Cu}-\mathrm{F}$ system contains an old puzzle of crystalline copper fluoride existence and synthesis, which produced a neverending debate and remained unresolved to date. The first report of synthesis of $\mathrm{CuF}$ with zincblende structure was published in 1933 [10]. It was then argued that the reported $\mathrm{CuF}$ is identical to $\mathrm{Cu}_{2} \mathrm{O}$, dismissing the previous experimental results [11]. Recently no one succeeded in reproducing the synthesis of $\mathrm{CuF}$, and the earliest studies have met strong criticism [12, 13], since it is commonly believed that fluorine, because of its high electronegativity, will always oxidize copper to the oxidation state +2 . Even though all attempts to synthesize $\mathrm{CuF}$ have been unsuccessful and the very existence of this compound is questionable, studies are ongoing [14, 15], and the complexes of $\mathrm{CuF}$ are already well characterized [16].

The computationally guided studies of new transition metal fluorides, and $\mathrm{CuF}$ in particular, also continue. Initially, they mainly compared different structure prototypes to find a hypothetical ground state crystal structure [17, 18] or investigated cluster formation [19]. A variety of new structures have been reported using evolutionary crystal structure prediction and assuming $\mathrm{CuF}$ stoichiometry 20]. On the basis of all previous studies, eventually, it has been shown that all predicted structures are metastable [18, 20].

Recently, a computational crystal structure prediction of coinage metal fluorides at different pressures was done [9]. However, the used method works with a fixed stoichiometry, which limits the prediction of new phases in the whole system. Moreover, redoing the same calculations lead to different structures [21]. Thus, the detailed and reliable analysis of the whole $\mathrm{Cu}-\mathrm{F}$ system remained to be done.

In this Letter, we present a first-principles variable- composition evolutionary crystal structure prediction study of all phases in the $\mathrm{CuF}$ system. We recover the experimentally known structure of $\mathrm{CuF}_{2}$ and report hitherto unknown stable $C 2 / m-\mathrm{Cu}_{2} \mathrm{~F}_{5}, R \overline{3} c-\mathrm{CuF}_{3}$, and Pnma- $\mathrm{CuF}_{3}$ phases. Based on the similarities between the crystal structure of the discovered fluorides and the structure of the parent cuprate high-temperature (high$T_{\text {c }}$ ) superconductor $\mathrm{La}_{2} \mathrm{CuO}_{4}$, we explored the possibility of high- $\mathrm{T}_{s}$ superconductivity in doped copper fluorides.

Stable phases in the $\mathrm{CuF}$ system were predicted here using the first-principles evolutionary algorithm as implemented in the USPEX package [22, 23]. The evolutionary search was combined with structure relaxation and energy calculations using density functional theory (DFT) within the PerdewBurkeErnzerhof (PBE) 24] exchangecorrelation functional and employing the projector augmented plane wave (PAW) method [25] as implemented in the VASP package [26]. We used the planewave energy cutoff of $600 \mathrm{eV}$ and $\Gamma$-centered $k$-meshes with a resolution of $2 \pi \times 0.05^{-1}$ for Brillouin zone sampling, ensuring excellent convergence of the quantities of interest. During the variable-composition structure search, the first generation of 160 structures was produced using random symmetric [27] and random topological [28] structure generators, with up to 18 atoms in the primitive cell. $70 \%$ of the next generation were obtained by applying variation operators (heredity, softmutation, lattice mutation) to the $70 \%$ of the lowest-energy structures of current generation and the other $30 \%$ of the generation were produced randomly.

Phases located on the thermodynamic convex hull are stable with respect to decomposition into elemental $\mathrm{Cu}$ and $\mathrm{F}$ or other $\mathrm{CuF}$ compounds. The spin-polarized DFT calculations lead to the convex hull diagram as presented on the (Fig. 1). It contains experimentally known $P 2_{1} / c^{-}$ $\mathrm{CuF}_{2}$, hitherto unknown $C 2 / m-\mathrm{Cu}_{2} \mathrm{~F}_{5}, R \overline{3} c-\mathrm{CuF}_{3}$, and slightly metastable $\mathrm{Pnma}-\mathrm{CuF}_{3}$, which is just $0.001 \mathrm{eV}$ above the convex hull. Successful prediction of the $\mathrm{CuF}_{2}$, a known compound, indicates the robustness of our methodology. All obtained potentially stable struc- 
tures became subject of an additional fixed-composition study taking into account up to four formula units (and up to 18 atoms in the unit cell for $\mathrm{CuF}$ ). The dynamical stability of all structures was carefully verified with phonon calculations using the supercell approach and the finite displacement method, as implemented in the Phonopy package [29]. The structural information for the obtained compounds and results of the phonon calculations are presented in the Supporting Materials (SM).

The most energetically favorable structure of $\mathrm{CuF}$, found in our study, is the low-symmetry $P 1-\mathrm{CuF}$, which is even lower in energy than previous reports [20] by $\sim 0.05 \mathrm{meV} /$ atom, but its low symmetry and high energy ( $\sim 50 \mathrm{meV} /$ atom above the convex hull) indicated its instability and tendency to decompose into $\mathrm{Cu}+\mathrm{CuF}_{2}$. Thus, we conclude $\mathrm{CuF}$ is unlikely to exist at ambient pressure.

$\mathrm{Cu}_{2} \mathrm{~F}_{5}$ crystallizes in the monoclinic space group $C 2 / m$ with two inequivalent $\mathrm{Cu}$ sites, where each $\mathrm{Cu}$ atom of the first type is bonded to six pairwise equivalent $\mathrm{F}$ atoms forming a $\mathrm{CuF}_{6}$ octahedron (Fig. 2a), with the cornersharing octahedral tilt angles of $0^{\circ}$. In the second site, the $\mathrm{Cu}$ atom is in a square planar geometry with four pairwise equivalent $\mathrm{F}$ atoms. This arrangement could be also described as a distorted octahedron (see SM Fig. 3a). While isostoichiometric $P \overline{1}-\mathrm{Ag}_{2} \mathrm{~F}_{5}$ is well-known [7], hypothetical $P \overline{1}-\mathrm{Cu}_{2} \mathrm{~F}_{5}$ has a higher energy than $C 2 / m$ $\mathrm{Cu}_{2} \mathrm{~F}_{5}$ by $\sim 3 \mathrm{meV} /$ atom in the spin-polarized DFT solution.

Ground state $\mathrm{CuF}_{3}$ has a trigonal perovskite structure with the space group $R \overline{3} c$ (Fig. 2b). This structure was also predicted in [9]. The $\mathrm{Cu}$ atom is bonded with six equivalent $\mathrm{F}$ atoms to form an octahedron with the corner-sharing octahedral tilt angles of $29^{\circ}$. Orthorhombic $P n m a-\mathrm{CuF}_{3}$, metastable at $0 \mathrm{~K}$, also has a perovskite structure $\mathrm{ABX}_{3}$ with absent $\mathrm{A}$ cations - $\mathrm{ReO}_{3}$-type structure (Fig. 2k), with the corner-sharing octahedral tilt angles of $28^{\circ}$. Metal trifluorides $\mathrm{FeF}_{3}, \mathrm{CoF}_{3}, \mathrm{RuF}_{3}$, $\mathrm{RhF}_{3} \mathrm{PdF}_{3}$, and $\mathrm{IrF}_{3}$ also have perovskite structure with the space group $R \overline{3} c$ [30], whereas $\mathrm{AgF}_{3}$ and $\mathrm{AuF}_{3}$ crystallize in a totally different structure with the space group $P 66_{1} 22$ [31, 32]. Hypothetical $P 6_{1} 22-\mathrm{CuF}_{3}$ has a higher energy than the $R \overline{3} c$ phase by $\sim 30 \mathrm{meV} /$ atom in the spin-polarized DFT solution. Notably, perovskite-type structures frequently have octahedral tilt instabilities and exhibit phase transition [33].

Discovered $\mathrm{Cu}$ fluorides have significant crystalchemical similarities with high- $\mathrm{T}_{s}$ cuprates. In both systems we observe $\mathrm{Cu}^{+2}$ (in square planar coordination, as a consequence of Jahn-Teller distortion in all cuprates and $\mathrm{Cu}_{2} \mathrm{~F}_{5}$ ) and $\mathrm{Cu}^{+3}$ (in $\mathrm{CuF}_{3}, \mathrm{Cu}_{2} \mathrm{~F}_{5}$, and in doped cuprates). As we discuss below, pure parent compounds $\mathrm{CuF}_{3}, \mathrm{Cu}_{2} \mathrm{~F}_{5}$, and $\mathrm{La}_{2} \mathrm{CuO}_{4}$ are antiferromagnetic insulators, but doping by electrons or holes makes them metallic and superconducting (for sure $\mathrm{La}_{2} \mathrm{CuO}_{4}$, and most likely for copper fluorides).

To compare the electronic properties of copper fluorides and cuprate, we firstly performed

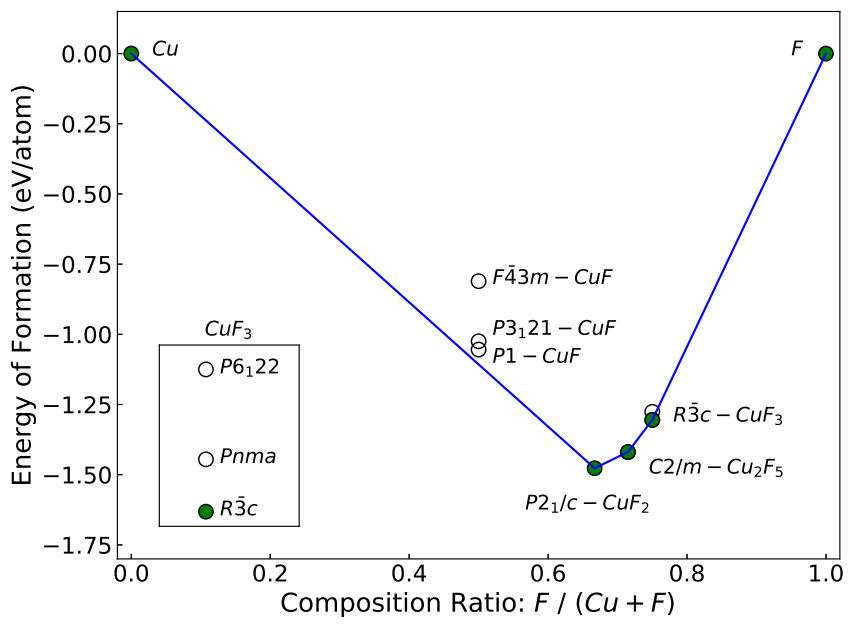

FIG. 1. Convex hull diagram of the CuF system. The inset schematically mentions existence of three $\mathrm{CuF}_{3}$ phases.

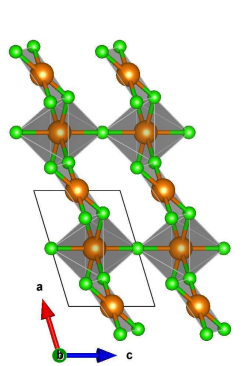

(a)

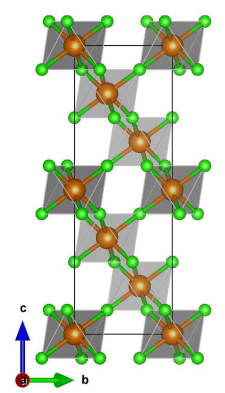

(b)

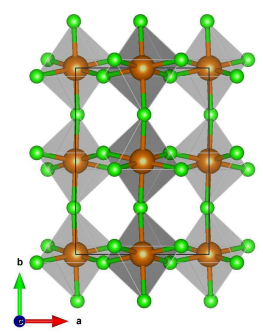

(c)
FIG. 2. (color online). Schematic representation of the crystal structures: (a) $C 2 / m-\mathrm{Cu}_{2} \mathrm{~F}_{5}$, (b) $R \overline{3} c-\mathrm{CuF}_{3}$, (c) Pnma$\mathrm{CuF}_{3}$. The $\mathrm{Cu}$ and $\mathrm{F}$ atoms are shown in brown and green, respectively. Structures were visualized using VESTA software 34.

the spin-unpolarized DFT calculations using dense MonkhorstPack meshes of $8 \times 8 \times 4$ and $12 \times 12 \times 12$ kpoints for distorted orthorombic low-temperature Bamb$\mathrm{La}_{2} \mathrm{CuO}_{4}$ phase and all fluorides, respectively. Structural information and energies for ferromagnetic and antiferromagnetic orders are presented in the (SM Tab.1, 2). The densities of states (DOS) for $R \overline{3} c-\mathrm{CuF}_{3}, \mathrm{Cu}_{2} \mathrm{~F}_{5}$, and $B a m b-\mathrm{La}_{2} \mathrm{CuO}_{4}$ resolved for the $\mathrm{Cu}-\mathrm{d}$ and ligand-p states within DFT are shown on (Fig. 3a, c, e). In all systems DFT results show that the $\mathrm{Cu}-3 \mathrm{~d}$ energy band is located completely inside the $\mathrm{p}$ band of the ligands and strongly hybridizes with it. Therefore, the partially filled electronic states of interest are formed by the d- and psymmetry states with approximately equal weights, and the usual ionic picture is not applicable for such a band structure. Notably, the magnetic exchange interaction is proportion to the scale of magnetic fluctuations. For all considered systems, solutions with antiferromagnetic order are the lowest in energy (SM Tab.3, 4). Since the energy difference obtained from the spin-unpolarized and spin-polarized DFT calculations is quite small, one can 
expect strong spin fluctuations in both types of systems and we recall that high- $\mathrm{T}_{c}$ superconductivity of cuprates is believed to be mediated by spin fluctuations. Doped $\mathrm{Cu}$ fluorides can, or perhaps, even should be superconducting by the same magnetically mediated mechanism.

Although DFT shade light on some premature analogy with cuprates, in principle, this method is pathological since cannot correctly reproduce the antiferromagnetic insulating state of $\mathrm{La}_{2} \mathrm{CuO}_{4}$ because it neglects on-site Coulomb correlations [35], and more robust results are achieved by taking into account the electronic correlations using the $\mathrm{DFT}+\mathrm{U}$ method with the Coulomb interaction parameter $U=8 \mathrm{eV}$ and the exchange interaction parameter $J=0.9 \mathrm{eV}[35,36]$. Because we deal with copper in the same divalent and trivalent states, and the energy bands in cuprates and copper fluorides studied here have similar widths (Fig. 3a, c, e and Tab. I), we chose the same values of $U$ and $J$ for all calculations taking into account the on-site Coulomb repulsion between the $\mathrm{Cu}-3 \mathrm{~d}$ electrons in $\mathrm{CuF}_{3}, \mathrm{Cu}_{2} \mathrm{~F}_{5}$, and $\mathrm{Bamb}-\mathrm{La}_{2} \mathrm{CuO}_{4}$. Structural information obtained after the relaxation with $\mathrm{DFT}+\mathrm{U}$ as well as the values of total energy for ferromagnetic and antiferromagnetic orders are presented in the (SM Tab.1, 2).

We reproduced the insulating antiferromagnetic ground state of $\mathrm{Bamb}-\mathrm{La}_{2} \mathrm{CuO}_{4}$ with the $\mathrm{DFT}+\mathrm{U}$ energy gap of about $2 \mathrm{eV}$ and the magnetic moment of the $\mathrm{Cu}$ atoms of $0.65 \mu_{\mathrm{B}}$, which is in a close agreement with the experimentally observed values of $\sim 2 \mathrm{eV}$ and $0.68 \mu_{\mathrm{B}}$, respectively [37]. The DOS for $R \overline{3} c-\mathrm{CuF}_{3}, \mathrm{Cu}_{2} \mathrm{~F}_{5}$, and $B a m b-\mathrm{La}_{2} \mathrm{CuO}_{4}$ obtained using $\mathrm{DFT}+\mathrm{U}$ are presented on (Fig. 3 b d, f). The DOS for Pnma phase is presented on the (Fig. 2a, b in SM). For $R \overline{3} c-\mathrm{CuF}_{3}$ and Pnma- $\mathrm{CuF}_{3}$ in the antiferromagnetic phase, the $\mathrm{DFT}+\mathrm{U}$ calculations show similarities in the key features of the electronic structures of $\mathrm{CuF}_{3}$ and $\mathrm{Bamb}-\mathrm{La}_{2} \mathrm{CuO}_{4}$ - they have well-separated Hubbard bands formed by the $\mathrm{Cu}$ $\mathrm{d}$ states, whereas the first ionization states have a psymmetry and are formed by the ligands.

In the $\mathrm{CuF}_{3}$ structures, copper has an atypical formal oxidation state of +3 , which leads to the $3 \mathrm{~d}^{8}$ electronic configuration, whereas in $\mathrm{Bamb}-\mathrm{La}_{2} \mathrm{CuO}_{4}$ there are $\mathrm{Cu}^{2+}$ JahnTeller active ions. However, some or all of $\mathrm{Cu}^{+3}$ ions in $\mathrm{CuF}_{3}$ can be reduced to $\mathrm{Cu}^{+2}$ by electron doping, like in the well-known perovskite-type $\mathrm{KCuF}_{3}$, where all $\mathrm{Cu}$ atoms are in the oxidation state $+2 . \mathrm{CuF}_{3}$, in fact, can be described as the structure of $\mathrm{KCuF}_{3}$ with all $\mathrm{K}$ atoms removed. Thus, one way to make a superconducting $\mathrm{Cu}$ fluoride is to remove part of $\mathrm{K}$ atoms from $\mathrm{KCuF}_{3}$ (in a vacuum tube) - the result should be a metallic perovskite-type compound with mixed $\mathrm{Cu}^{+2}$ and $\mathrm{Cu}^{+3}$ states. To clearly show this, we performed a fixed-composition structure search of $\mathrm{K}_{3}\left(\mathrm{CuF}_{3}\right)_{4}$, which determined that the most stable phase has perovskitetype structure with the space group $\operatorname{Im} \overline{3} m$ (SM Fig. 3c). This structure is stable with respect to the decomposition into $R \overline{3} c-\mathrm{CuF}_{3}$ and $\mathrm{KCuF}_{3}(\sim 0.05 \mathrm{eV} /$ atom below the decomposition line), which means that potassium ions

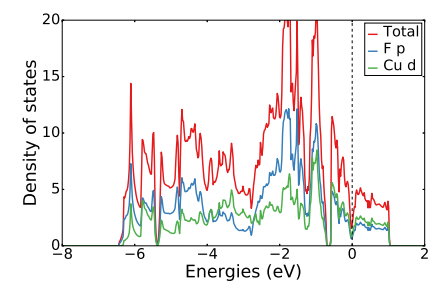

(a)

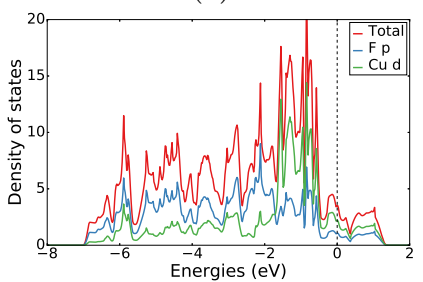

(c)

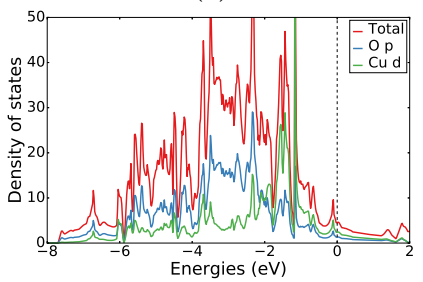

(e)

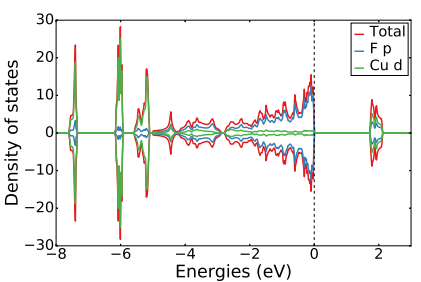

(b)

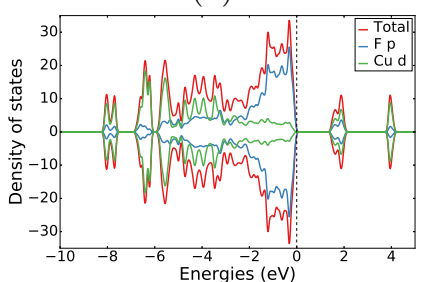

(d)

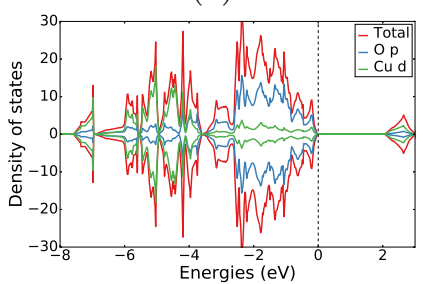

(f)
FIG. 3. (color online). Total and partial density of states for (a, b) $R \overline{3} c-\mathrm{CuF}_{3}$, (c, d) $\mathrm{Cu}_{2} \mathrm{~F}_{5}$, and (e, f) $B a m b-\mathrm{La}_{2} \mathrm{CuO}_{4}$ obtained using (a, c, e) DFT and (b, d, f) DFT+U.

can be easily extracted from the $\mathrm{KCuF}_{3}$, forming mixedvalence compound.

TABLE I. Bandwidth $W$ and charge transfer gap $\Delta_{\text {pd }}$ calculated using DFT. Hubbard bands splitting $U_{\mathrm{dd}}$, spin $S$, and magnetic moment $M$ obtained using the DFT+U method. The values in parentheses are related to the second type of $\mathrm{Cu}$ atoms in the $\mathrm{Cu}_{2} \mathrm{~F}_{5}$.

\begin{tabular}{l|ccccc}
\hline \hline & $W(\mathrm{eV})$ & $\Delta_{\mathrm{pd}}(\mathrm{eV})$ & $U_{\mathrm{dd}}(\mathrm{eV})$ & $S$ & $M\left(\mu_{\mathrm{B}}\right)$ \\
\hline$R 3 c-\mathrm{CuF}_{3}$ & 8 & 1.7 & 9.5 & 1 & 1.15 \\
$\mathrm{Cu}_{2} \mathrm{~F}_{5}$ & 8 & 1.42 & 9.5 & $1(1 / 2)$ & $1.17(0.79)$ \\
$\mathrm{La}_{2} \mathrm{CuO}_{4}$ & 9 & 2 & 10.5 & $1 / 2$ & 0.65 \\
\hline \hline
\end{tabular}

According to the $\mathrm{DFT}+\mathrm{U}$ solution: each $\mathrm{Cu}$ site in the $\mathrm{CuF}_{3}$ has spin $1 ; \mathrm{Cu}_{2} \mathrm{~F}_{5}$ is determined as a compound with the mixed-valence $\mathrm{Cu}^{2+} / \mathrm{Cu}^{3+}$ state and $\mathrm{Cu}$ ions with spin 1 and $1 / 2$; $\mathrm{Cu}$ ions in the cuprate have spin $1 / 2$. The magnetic moments per $\mathrm{Cu}$ atom obtained in the $\mathrm{DFT}+\mathrm{U}$ calculations for $R \overline{3} c-\mathrm{CuF}_{3}$ are $1.15 \mu_{\mathrm{B}}\left(1.14 \mu_{\mathrm{B}}\right.$ in the Pnma phase). These magnetic moment values are smaller by a factor of 0.58 than the formal ionic value of $2 \mu_{\mathrm{B}}$ for a $\mathrm{Cu}^{3+}$ ion compared to the reduction factor of 0.65 for the formal atomic value of $1 \mu_{\mathrm{B}}$ for a $\mathrm{Cu}^{2+}$ ion in the $\mathrm{La}_{2} \mathrm{CuO}_{4}$ [37]. For $\mathrm{Cu}_{2} \mathrm{~F}_{5}$ we found that two types of $\mathrm{Cu}$ atoms, have different formal electronic configurations, $\mathrm{d}^{8}$ and $\mathrm{d}^{9}$, and different magnetic moments of $1.17 \mu_{\mathrm{B}}$ and $0.79 \mu_{\mathrm{B}}$. All predicted copper fluoride structures and $\mathrm{Bamb}-\mathrm{La}_{2} \mathrm{CuO}_{4}$ are charge-transfer insulators 


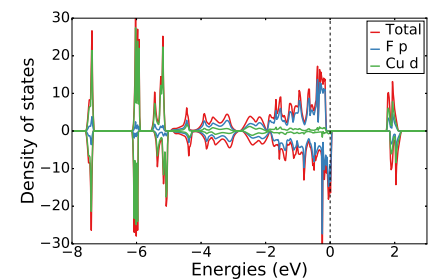

(a)

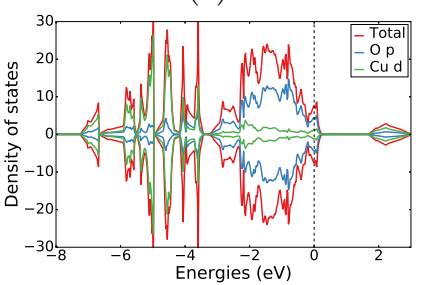

(c)

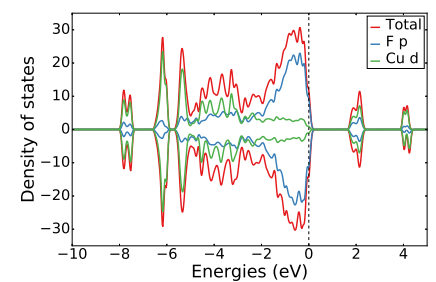

(b)

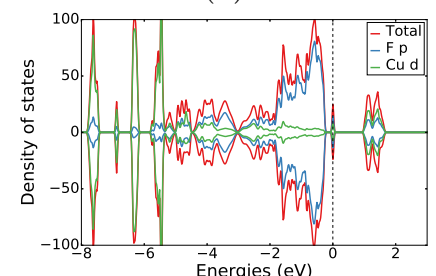

(d)

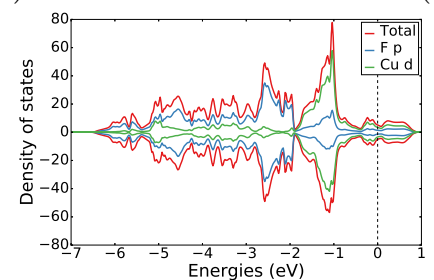

(e)

FIG. 4. (color online). Total and partial density of states for p-doped (a) $R \overline{3} c-\mathrm{CuF}_{3}$, (b) $\mathrm{Cu}_{2} \mathrm{~F}_{5}$, and (c) $\mathrm{La}_{2} \mathrm{CuO}_{4}$. (d) $R \overline{3} c-\mathrm{CuF}_{3}$ with the one $\mathrm{F}$ vacancy per $2 \times 2 \times 2$ supercell, (e) and perovskite-type $\operatorname{Im} \overline{3} \mathrm{~m}-\mathrm{K}_{3}\left(\mathrm{CuF}_{3}\right)_{4}$.

with respect to the classification of Zaanen et al. [38]. The first energy excitation occurs between the p band of the ligands and the $\mathrm{d}$ band of the metal ion. $\mathrm{CuF}_{3}$ has a small charge transfer gap (an important characteristic of cuprates) $\Delta_{\mathrm{pd}}=1.7 \mathrm{eV}, \mathrm{Cu}_{2} \mathrm{~F}_{5}$ also has a small charge transfer gap $\Delta_{\mathrm{pd}}=1.42 \mathrm{eV}$, comparable with $2 \mathrm{eV}$ of $\mathrm{La}_{2} \mathrm{CuO}_{4}$. Though the energy gap depends on the choice of the Hubbard $U$ parameter, the charge-transfer nature of the gap remains the same for a wide range of $U$ values in fluorides. The splitting between the Hubbard bands for $\mathrm{CuF}_{3}$ and $\mathrm{La}_{2} \mathrm{CuO}_{4}$ is similar and equals to $\sim 9.5 \mathrm{eV}$ and $10.5 \mathrm{eV}$, respectively (Fig. 3b, d).

$\mathrm{Cu}_{2} \mathrm{~F}_{5}, \mathrm{CuF}_{3}$, and $\mathrm{La}_{2} \mathrm{CuO}_{4}$ are insulators and can display superconductivity only when properly doped. Consequently, we performed DFT $+\mathrm{U}$ calculations of the considered systems doped with holes using rigid-band shift approximation, with doping amounted to 0.25 holes for each copper atom in the unit cell (Fig. 4a, b, c). $\mathrm{CuF}_{3}$ and $\mathrm{Cu}_{2} \mathrm{~F}_{5}$, like $\mathrm{La}_{2} \mathrm{CuO}_{4}$, undergo a transition from the insulating to the conducting state upon the hole doping, which once again highlights the similarity of their electronic properties. We have also examined a $2 \times 2 \times 2$ $R \overline{3} c$-CuF3 supercell (64 atoms) with a vacancy on one of the $\mathrm{F}$ atoms. This ferrimagnetic structure lies on the thermodynamic convex hull (Fig. 1). This means that the formation of the non-stoichiometric $\mathrm{CuF}_{3-x}$ is favorable. The DFT $+\mathrm{U}$ solution determines the formation of a peak at the Fermi level for this structure (Fig. 4 d). Perovskite-type $I m \overline{3} m-\mathrm{K}_{3}\left(\mathrm{CuF}_{3}\right)_{4}$ also has a metallic so-

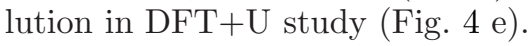

In summary, the results of the systematic crystal structure search in the $\mathrm{CuF}$ system supports that $\mathrm{CuF}$ is unlikely to exist and have revealed hitherto unknown $C 2 / \mathrm{m}$ $\mathrm{Cu}_{2} \mathrm{~F}_{5}, R \overline{3} c-\mathrm{CuF}_{3}$, and slightly metastable $P n m a-\mathrm{CuF}_{3}$. $\mathrm{Cu}_{2} \mathrm{~F}_{5}$ contains $\mathrm{Cu}$ ions with oxidation states +2 and +3 , which leads to the presence of two magnetic subsystems. In $\mathrm{CuF}_{3}, \mathrm{Cu}$ ions have an unusual oxidation state +3 , which can be reduced to +2 by proper doping. We showed that potassium can be extracted from $\mathrm{KCuF}_{3}$ forming metallic state. we showed using $\mathrm{DFT}+\mathrm{U}$ that all discovered copper fluorides are strongly correlated compounds and charge-transfer insulators. Since comparison of the $\mathrm{CuF}_{3}$ and $\mathrm{Cu}_{2} \mathrm{~F}_{5}$ with the classical cuprate $\mathrm{La}_{2} \mathrm{CuO}_{4}$ shows many similarities, discovered structures possibly could be a new class of high- $T_{\mathrm{c}}$ superconductors.

Acknowledgments. This work was supported by the Russian Science Foundation (Project 19-72-30043). Calculations were performed on Arkuda cluster of Skoltech and Uran cluster of IMM UB RAS. DYN, DMK and VIA thanks the Ministry of Science and Higher Education of the Russian Federation (No. AAAA-A18-1180201900985, topic "Electron").
[1] J. M. Winfield, Journal of Fluorine Chemistry 33, 159 (1986)

[2] J. S. Thrasher and S. H. Strauss, "Inorganic Fluorine Chemistry," in Inorganic Fluorine Chemistry (1994) Chap. 1, pp. 1-23.

[3] F. Wang, R. Robert, N. A. Chernova, N. Pereira, F. Omenya, F. Badway, X. Hua, M. Ruotolo, R. Zhang, L. Wu, V. Volkov, D. Su, B. Key, M. Stanley Whittingham, C. P. Grey, G. G. Amatucci, Y. Zhu, and J. Graetz, Journal of the American Chemical Society 133, 18828 (2011)

[4] F. Wang, H. C. Yu, M. H. Chen, L. Wu, N. Pereira, K. Thornton, A. Van Der Ven, Y. Zhu, G. G. Amatucci, and J. Graetz, Nature Communications 3, 1 (2012)

[5] X. Hua, R. Robert, L.-S. Du, K. M. Wiaderek, M. Leskes, K. W. Chapman, P. J. Chupas, and C. P. Grey,
The Journal of Physical Chemistry C 118, 15169 (2014)

[6] F. Omenya, N. J. Zagarella, J. Rana, H. Zhang, C. Siu, H. Zhou, B. Wen, N. A. Chernova, L. F. J. Piper, G. Zhou, and M. S. Whittingham, ACS Applied Energy Materials 2, 5243 (2019).

[7] W. Grochala and R. Hoffmann, Angewandte Chemie International Edition 40, 2742 (2001)

[8] J. Gawraczynski, D. Kurzydłowski, R. A. Ewings, S. Bandaru, W. Gadomski, Z. Mazej, G. Ruani, I. Bergenti, T. Jaron, A. Ozarowski, S. Hill, P. J. Leszczynski, K. Tokár, M. Derzsi, P. Barone, K. Wohlfeld, J. Lorenzana, and W. Grochala, Proceedings of the National Academy of Sciences of the United Stat [9] J. Lin, S. Zhang, W. Guan, G. Yang, and Y. Ma, 
Journal of the American Chemical Society 140, 9545 (2018) [23] A. R. Oganov, A. O. Lyakhov, and M. Valle,

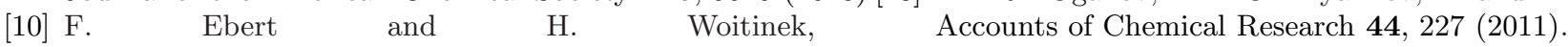
Zeitschrift für anorganische und allgemeine Chemie 210, 269[24933). P. Perdew, K. Burke, and M. Ernzerhof,

[11] H. M. Haendler, L. H. Towle, E. F. Bennett, and W. L. Patterson, Journal of the American Chemical Society 76, 2178 (1954).

[12] C. Housecroft and A. Sharpe, Inorganic Chemistry (Pearson Prentice Hall, 2005).

[13] N. N. Greenwood and A. Earnshaw, Chemistry of the Elements (Elsevier Science, 2012).

[14] X. Wang, L. Andrews, F. Brosi, and S. Riedel, Chemistry A European Journal 19, 1397 (2013).

[15] P. Woidy, A. J. Karttunen, M. Widenmeyer, R. Niewa, and F. Kraus, Chemistry A European Journal 21, 3290 (2015).

[16] D. J. Gulliver, W. Levason, and M. Webster, Inorganica Chimica Acta 52, 153 (1981).

[17] T. Söhnel, H. Hermann, and P. Schwerdtfeger, Journal of Physical Chemistry B 109, 526 (2005).

[18] A. Walsh, C. R. A. Catlow, R. Galvelis, D. O. Scanlon, F. Schiffmann, A. A. Sokol, and S. M. Woodley, Chemical Science 3, 2565 (2012).

[19] R. P. Krawczyk, A. Hammerl, and P. Schwerdtfeger, ChemPhysChem 7, 2286 (2006).

[20] M. S. Kuklin, L. Maschio, D. Usvyat, F. Kraus, and A. J. Karttunen, Chemistry A European Journal 25, 11528 (2019).

[21] G. Liu, X. Feng, L. Wang, S. A. Redfern, X. Yong, G. Gao, and H. Liu, Physical Chemistry Chemical Physics 21, 17621 (2019).

[22] A. R. Oganov and C. W. Glass, The Journal of Chemical Physics 124, 244704 (2006)

Physical Review Letters 77, 3865 (1996)

[25] P. E. Blöchl, Physical Review B 50, 17953 (1994)

[26] G. Kresse and J. Furthmüller, Physical Review B 54, 11169 (1996).

[27] A. O. Lyakhov, A. R. Oganov, H. T. Stokes, and Q. Zhu, Computer Physics Communications 184, 1172 (2013).

[28] P. V. Bushlanov, V. A. Blatov, and A. R. Oganov, Computer Physics Communications 236, 1 (2019).

[29] Scripta Materialia 108, 1 (2015).

[30] M. A. Hepworth, K. H. Jack, R. D. Peacock, and G. J. Westland, Acta Crystallographica 10, 63 (1957).

[31] B. Žemva, K. Lutar, A. Jesih, W. J. Casteel, A. P. Wilkinson, D. E. Cox, B. Robert von Dreele, H. Borrmann, and N. Bartlett, Journal of the American Chemical Society 113, 4192 (1991)

[32] F. W. Einstein, P. R. Rao, J. Trotter, and N. Bartlett, Journal of the Chemical Society A: Inorganic, Physical, and Theoret

[33] P. M. Woodward, Acta Crystallographica Section B 53, 32 (1997),

[34] K. Momma and F. Izumi, Journal of Applied Crystallography 41, 653 (2008)

[35] M. T. Czyyk and G. A. Sawatzky, Physical Review B 49, 14211 (1994).

[36] V. I. Anisimov, F. Aryasetiawan, and A. I. Lichtenstein, Journal of Physics Condensed Matter 9, 767 (1997)

[37] W. E. Pickett, Reviews of Modern Physics 61, 433 (1989)

[38] J. Zaanen, G. A. Sawatzky, and J. W. Allen, Physical Review Letters 55, 418 (1985). 